\title{
REMARKS ON COMPONENT FACTORS IN GRAPHS
}

\author{
GuOWEI DAI*10
}

\begin{abstract}
For a family of connected graphs $\mathcal{F}$, a spanning subgraph $H$ of a graph $G$ is called an $\mathcal{F}$-factor of $G$ if its each component is isomorphic to an element of $\mathcal{F}$. In particular, $H$ is called an $\mathcal{S}_{k}$-factor of $G$ if $\mathcal{F}=\left\{K_{1,1}, K_{1,2}, \ldots, K_{1, k}\right\}$, where integer $k \geq 2 ; H$ is called a $P_{\geq 3}$-factor of $G$ if every component in $\mathcal{F}$ is a path of order at least three. As an extension of $\mathcal{S}_{k}$-factors, the induced star-factor (i.e., $\mathcal{I S}_{k}$-factor) is a spanning subgraph each component of which is an induced subgraph isomorphic to some graph in $\mathcal{F}=\left\{K_{1,1}, K_{1,2}, \ldots, K_{1, k}\right\}$. In this paper, we firstly prove that a graph $G$ has an $\mathcal{S}_{k}$-factor if and only if its isolated toughness $I(G) \geq \frac{1}{k}$. Secondly, we prove that a planar graphs $G$ has an $\mathcal{S}_{2}$-factors if its minimum degree $\delta(G) \geq 3$. Thirdly, we give two sufficient conditions for graphs with $\mathcal{I} \mathcal{S}_{k}$-factors by toughness and minimum degree, respectively. Additionally, we obtain three special classes of graphs admitting $P_{\geq 3}$-factors.
\end{abstract}

Mathematics Subject Classification. 05C70, 05C38.

Received September 16, 2021. Accepted February 27, 2022.

\section{INTRODUCTION}

The graphs considered here are finite and simple, unless explicitly stated. Let $G=(V(G), E(G))$ be a graph. We denote by $V(G)$ and $E(G)$ the vertex set and the edge set of $G$, respectively. For $v \in V(G)$, we use $d_{G}(v)$ and $N_{G}(v)$ to denote the degree of $v$ and the set of vertices adjacent to $v$ in $G$, respectively. For $S \subseteq V(G)$, we write $N_{G}(S)=\cup_{v \in S} N_{G}(v)$. A graph $G$ is called an $r$-regular graph if $d_{G}(v)=r$ for each $v \in V(G)$. We use $\delta(G)$ to denote the minimum degree of a graph $G$. The number of connected components and isolated vertices of a graph $G$ is denoted by $\omega(G)$ and $i(G)$, respectively. We refer to [3] for the notation and terminologies not defined here.

The complete bipartite graph $K_{1, r}$ is called the star of order $r+1$, where $r$ is a positive integer. We use $\mathcal{S}_{k}$ to denote the set $\left\{K_{1,1}, K_{1,2}, K_{1,3}, \ldots, K_{1, k}\right\}$, where integer $k \geq 2$.

Let $\mathcal{F}$ be a family of connected graphs. Then a spanning subgraph $H$ of $G$ is called an $\mathcal{F}$-factor of $G$ if each component of $H$ is isomorphic to an element of $\mathcal{F}$. In particular, for an integer $k \geq 2$, a $\left\{K_{1,1}, K_{1,2}, K_{1,3}, \ldots, K_{1, k}\right\}$-factor is briefly called an $\mathcal{S}_{k}$-factor. Similarly, a $\left\{P_{k}, P_{k+1}, \ldots\right\}$-factor is called a $P_{\geq k}$-factor.

In 1947, Tutte [10] presented a criterion for the existence of 1-factors (perfect matchings), which is one of the classical results in graph theory. Denote by $o(G)$ the number of odd components of $G$, whose orders are odd.

Keywords. Star-factor, Induced star-factor, $P_{\geq 3}$-factor, Toughness, Minimum degree.

1 School of Mathematical Sciences, Nanjing Normal University, Nanjing, Jiangsu 210023, P.R. China.

*Corresponding author: guowei_dai@aliyun.com 
Theorem 1.1. (Tutte [10]) $A$ graph $G$ has a 1-factor if and only if $o(G-S) \leq|S|$ for any $S \subseteq V(G)$.

Since the well-known Tutte 1-factor theorem [10] was proposed, there are many results about componentfactors, see $[5,9,13,14]$, etc.

Akiyama, Avis and Era [1] demonstrated the following classical result, which is a characterization for the existence of $P_{\geq 2}$-factors in a graph.

Theorem 1.2. (Akiyama, Avis and Era [1]) A graph $G$ has a $P_{\geq 2}$-factor if and only if $i(G-S) \leq 2|S|$ for any $S \subseteq V(G)$.

Amahashi and Kano [2] and Las Vergnas [11] gave independently a characterization for graphs with $\mathcal{S}_{k}$-factors, which is a generalization of Theorem 1.2.

Theorem 1.3. (Amahashi and Kano [2]; Las Vergnas [11]) Let $k$ be an integer with $k \geq 2$. Then a graph $G$ has an $\mathcal{S}_{k}$-factor if and only if $i(G-S) \leq k|S|$ for any $S \subseteq V(G)$.

A connected graph is called a cactus if each block of the graph is a complete subgraph. A cactus of odd order is called an odd-cactus. As an extension of $\mathcal{S}_{k}$-factors, the induced star factor, denoted by $\mathcal{I S}_{k}$-factor, is a spanning subgraph each component of which is an induced subgraph isomorphic to some graph in $\left\{K_{1,1}, K_{1,2}, \ldots, K_{1, k}\right\}$. Denote by $o c(G-S)$ the number of odd-cactus of $G-S$. The criterion for $\mathcal{I S}_{k}$-factors was obtained by Egawa, Kano and Kelmans as following.

Theorem 1.4. (Egawa, Kano and Kelmans [6]) Let $k \geq 2$ be an integer. A graph $G$ has an $\mathcal{I S}_{k}$-factor if and only if oc $(G-S) \leq k|S|$ for any $S \subseteq V(G)$.

The toughness of a connected graph $G$, denoted by $\tau(G)$, was first introduced by Chvátal [4] as follows. If $G$ is complete, then $\tau(G)=+\infty$; otherwise,

$$
\tau(G)=\min \left\{\frac{|S|}{\omega(G-S)}: S \subseteq V(G), \omega(G-S) \geq 2\right\}
$$

Kaneko [7] introduced the concept of a sun and gave a characterization for the existence of $P_{\geq 3}$-factors in a graph. It is perhaps the first criterion of graphs admitting path factors not including $P_{2}$. Additionally, Kano et al. [8] obtained a simpler proof for Kaneko's result [7].

A graph $H$ is said to be a factor-critical graph if for each $v \in V(H), H-\{v\}$ has a 1-factor. Let $H$ be a factor-critical graph such that $V(H)=\left\{v_{1}, v_{2}, \ldots, v_{n}\right\}$. A graph is called a sun if it is obtained from $H$ by adding new vertices $\left\{u_{1}, u_{2}, \ldots, u_{n}\right\}$ together with new edges $\left\{v_{i} u_{i}: 1 \leq i \leq n\right\}$ to $H$. Note that, according to Kaneko [7], $K_{1}$ and $K_{2}$ are also regarded as a sun, respectively. Usually, the suns other than $K_{1}$ are called big suns. We use $\operatorname{sun}(G-X)$ to denote the number of sun components of $G-X$.

Theorem 1.5. (Kaneko [7]) $A$ graph $G$ has a $P_{\geq 3}$-factor if and only if $\operatorname{sun}(G-S) \leq 2|S|$ for any $S \subseteq V(G)$.

Corollary 1.6. (Kaneko [7]) $A$ graph $G$ has a $P_{\geq 3}$-factor if one of the following holds: (i) $G$ is $r$-regular where $r \geq 2$; (ii) $\tau(G)=1$; (iii) $\tau(G)=\frac{1}{2}$ and $\delta(G) \geq 2$; (iv) $G$ is 3-connected planar; (v) $G$ is claw-free with $\delta(G) \geq 2$.

This paper attempts to find more sufficient conditions for the existence of these component factors by different graphic parameters including minimum degree, toughness, isolated toughness, binding number, etc. 


\section{STAR-FACTOR}

The isolated toughness of a connected graph $G$ denoted by $I(G)$. If $G$ is complete, then $I(G)=+\infty$; otherwise,

$$
I(G)=\min \left\{\frac{|S|}{i(G-S)}: S \subseteq V(G), i(G-S) \geq 2\right\} .
$$

Lemma 2.1. [15] Let $G$ be a graph and $k \geq 1$ be a real number. Then the following three statements are equivalent.

(i) $i(G-S) \leq k|S|$ for all $S \subset V(G)$.

(ii) $|U| \leq k\left|N_{G}(U)\right|$ for all independent set $U$ of $G$.

Theorem 2.2. A connected nontrivial graph $G$ has an $\mathcal{S}_{k}$-factor if and only if $I(G) \geq \frac{1}{k}$, where integer $k \geq 2$.

Proof. Sufficiency: If $G$ is complete and nontrivial, then $G$ has an $\mathcal{S}_{k}$-factor obviously. Thus we may assume that $G$ is a graph of order at least two and not complete. Suppose, by way of contradiction, that $G$ has no $\mathcal{S}_{k}$-factor, then by Theorem 1.3, there is a subset $S \subseteq V(G)$ such that $i(G-S)>k|S|$. Then, by the integrality of $i(G-S)$, we obtain that

$$
i(G-S) \geq k|S|+1 .
$$

If $|S|=0$, then $i(G)=i(G-S) \geq k|S|+1=1$, which contradicts the fact that $G$ is connected.

If $|S|=1$, then $i(G-S)>k|S|=k$. By the definition of $I(G)$, we have that

$$
I(G) \leq \frac{|S|}{i(G-S)}<\frac{1}{k},
$$

a contradiction.

If $|S| \geq 2$, then by $(2.1)$, we have

$$
|S| \leq \frac{i(G-S)-1}{k}
$$

By the definition of $I(G)$, we have

$$
\begin{aligned}
I(G) & \leq \frac{|S|}{i(G-S)} \\
& \leq \frac{i(G-S)-1}{k \times i(G-S)} \\
& =\frac{1}{k}-\frac{1}{k \times i(G-S)} \\
& <\frac{1}{k},
\end{aligned}
$$

a contradiction.

Necessity: Suppose that $G$ has an $\mathcal{S}_{k}$-factor and $I(G)<\frac{1}{k}$. Then by Theorem 1.3 and Lemma 2.1, for each independent set $U \subseteq V(G)$, we have

$$
|U| \leq k\left|N_{G}(U)\right| .
$$

Since $I(G)<\frac{1}{k}$, there is a subset $S \subseteq V(G)$ such that $\frac{|S|}{i(G-S)}<\frac{1}{k}$. Let $U$ be the set of isolated vertices of $G-S$, then $N_{G}(U) \subseteq S$. Obviously, $U$ is independent and

$$
\left|N_{G}(U)\right| \leq|S|<\frac{i(G-S)}{k}=\frac{|U|}{k},
$$

which contradicts $(2.2)$. 
Lemma 2.3. [3] Let $G$ be a simple connected planar graph of order at least three. If $G$ does not contain triangles, then $|E(G)| \leq 2|V(G)|-4$.

Theorem 2.4. Let $G$ be a connected planar graph. If $\delta(G) \geq 3$, then $G$ has an $\mathcal{S}_{2}$-factor.

Proof. Suppose that $G$ is a connected planar graph with no $\mathcal{S}_{2}$-factor. By Theorem 1.3, there exists a subset $S \subseteq V(G)$ such that $i(G-S)>2|S|$. According to the integrality of $i(G-S)$, we obtain that

$$
i(G-S) \geq 2|S|+1
$$

Claim 2.5. $S \neq \emptyset$.

Proof. Suppose $S=\emptyset$, by (2.3), $i(G-S) \geq 2|S|+1=1$. On the other hand, $i(G) \leq \omega(G)=1$ since $G$ is a connected graph. So, we obtain that $G$ is an isolated vertex, which contradicts that $\delta(G) \geq 3$.

By Claim 2.5, $S \neq \emptyset$. Set $|S|=s$. Then by $(2.3), i(G-S) \geq 2 s+1$. The set of isolated vertices in $G-S$ is denoted by $I(G-S)$. Then we construct a simple bipartite graph $H=H[X, Y]$ as follows. Let $X=S$ and $Y \subseteq I(G-S)$ such that $|Y|=2 s+1$. For any $s \in X$ and $y \in Y, s y \in E(H)$ if and only if $s y \in E(G)$. Since $\delta(G) \geq 3$, it is clear that for each $y \in Y$, we have $\left|N_{H}(y)\right| \geq 3$. Hence, $|H|=s+(2 s+1)=3 s+1 \geq 4$ and

$$
|E(H)| \geq 3 \times(2 s+1)=6 s+3>6 s .
$$

As $G$ is a connected planar graph, it is easy to see that $H$ is also a connected planar graph. According to the fact that a bipartite graph does not contain any odd cycles, Lemma 2.3 implies that

$$
\begin{aligned}
|E(H)| & \leq 2|H|-4 \\
& =2 \times(3 s+1)-4 \\
& =6 s-2 \\
& <6 s
\end{aligned}
$$

which is a contradiction to (2.4).

Remark 2.6. Now, we explain that the condition of minimum degree $\delta(G) \geq 3$ in Theorem 2.4 is the best possible. Let $G=2 K_{1} \vee 5 K_{1}$ be a complete bipartite graph, where $\vee$ means "join". We know that $G$ is a connected planar graph with $\delta(G)=2<3$. Choose $X=: V\left(2 K_{1}\right)$ with $|X|=2$, then we have that

$$
i(G-X)=5>2|X|=4 .
$$

In view of Theorem $1.3, G$ has no $\mathcal{S}_{2}$-factor.

\section{INDUCED STAR-FACTOR}

Theorem 3.1. Let $G$ be a connected graph of order at least three. If $G$ is not an odd cactus and $\tau(G) \geq \frac{1}{k}$, then $G$ has an $\mathcal{I S}_{k}$-factor.

Proof. Suppose, to the contrary, that $G$ is a connected graph with no $\mathcal{I} \mathcal{S}_{k}$-factor. If $G$ is a complete graph, then $G$ has a Hamilton cycle, denoted by $C$. Since $G$ is not an odd cactus, $C$ is an even cycle and thus $G$ has a 1-factor. Hence, $G$ has an $\mathcal{I} \mathcal{S}_{k}$-factor, a contradiction. Thus, we may assume that $G$ is not a complete graph.

By Theorem 1.4, there is a subset $S \subseteq V(G)$ such that $o c(G-S)>k|S|$. Due to the integrality, we obtain

$$
o c(G-S) \geq k|S|+1 .
$$


Claim 3.2. $S \neq \emptyset$.

Proof. Suppose that $S=\emptyset$, then by (3.1), we have $o c(G)=o c(G-S) \geq k|S|+1=1$. Note that $o c(G) \leq \omega(G)=1$ since $G$ is connected. Thus $G$ is an odd cactus, a contradiction.

By Claim 3.2, we have $|S| \geq 1$.

If $|S|=1$, then by (3.1), we have $o c(G-S) \geq k|S|+1=k+1$. Then due to the definition of $\tau(G)$, we obtain that

$$
\frac{1}{k} \leq \tau(G) \leq \frac{|S|}{\omega(G-S)} \leq \frac{|S|}{o c(G-S)} \leq \frac{1}{k+1}<\frac{1}{k}
$$

a contradiction.

If $|S| \geq 2$, then by (3.1), we have

$$
|S| \leq \frac{o c(G-S)-1}{k}
$$

Then by (3.2) and the definition of $\tau(G)$, we obtain that

$$
\begin{aligned}
\frac{1}{k} \leq \tau(G) & \leq \frac{|S|}{\omega(G-S)} \\
& \leq \frac{|S|}{o c(G-S)} \\
& \leq \frac{o c(G-S)-1}{k \times o c(G-S)} \\
& =\frac{1}{k}-\frac{1}{k \times o c(G-S)}<\frac{1}{k}
\end{aligned}
$$

a contradiction.

Theorem 3.3. Let $G$ be a connected graph of order $n \geq 3$ which is not an odd cactus. Then $G$ has an $\mathcal{I} \mathcal{S}_{k}$-factor if $\delta(G) \geq \max \left\{\frac{n}{k+1}, \frac{4 n}{3 k+1}-1\right\}$.

Proof. Suppose, to the contrary, that $G$ is a connected graph having no $\mathcal{I} \mathcal{S}_{k}$-factor. By Theorem 1.4, there exists $S \subseteq V(G)$ such that $o c(G-S)>k|S|$. Due to the integrality, we obtain

$$
o c(G-S) \geq k|S|+1 .
$$

Claim 3.4. $S \neq \emptyset$.

Proof. Suppose that $S=\emptyset$, then by (3.3), we have $o c(G)=o c(G-S) \geq k|S|+1=1$. Note that $o c(G) \leq \omega(G)=1$ since $G$ is connected. Thus $G$ is an odd cactus, a contradiction.

By Claim 3.4 and (3.3), we have that

$$
o c(G-S) \geq k|S|+1 \geq k+1 .
$$

Let $C_{1}, C_{2}, \ldots, C_{m}$ be the odd cactus components of $G-S$, where $m=o c(G-S)$. Choose an odd cactus component $C_{i}$ of $G-S$ such that $\left|C_{i}\right|$ is as small as possible, where $1 \leq i \leq m$. Without loss of generality, we assume that $C_{1}$ is such an odd cactus component and $\left|C_{1}\right|=t$. 
Case 1. $t=1$.

In this case, let $C_{1}=\{x\}$. Since $N_{G}(x) \subseteq S$, we have that

$$
|S| \geq d_{G}(x) \geq \delta(G) \geq \frac{n}{k+1} .
$$

It follows from (3.3) that

$$
\begin{aligned}
|G| & \geq|S|+\sum_{i=1}^{m}\left|C_{i}\right| \\
& \geq|S|+(k|S|+1) \\
& =(k+1)|S|+1 \\
& \geq(k+1) \times \frac{n}{k+1}+1 \\
& =n+1,
\end{aligned}
$$

a contradiction.

Case 2. $t \geq 2$.

Since $C_{1}$ is an odd cactus and $t \geq 2$, we find that $\left|C_{1}\right|=t \geq 3$. On the other hand, according to the minimality property, we have that

$$
t \leq \frac{|G|}{o c(G-S)} \leq \frac{n}{k|S|+1} \leq \frac{n}{k+1}<\frac{3 n}{3 k+1} .
$$

Let $u$ be the vertex with maximum degree in $C_{1}$, then $d_{C_{1}}(u) \leq t-1$. It follows that

$$
\begin{aligned}
|S| & \geq d_{S}(u) \\
& \geq \delta(G)-d_{C_{1}}(u) \\
& \geq \frac{4 n}{3 k+1}-1-(t-1) \\
& =\frac{4 n}{3 k+1}-t .
\end{aligned}
$$

This together with (3.3), (3.5) and $t \geq 3$ implies that

$$
\begin{aligned}
|G| & \geq|S|+\sum_{i=1}^{m}\left|C_{i}\right| \\
& \geq|S|+(k|S|+1) \times t \\
& >(k t+1)|S| \\
& \geq(k t+1) \times\left(\frac{4 n}{3 k+1}-t\right) \\
& =(k t+1) \times\left(\frac{n}{3 k+1}+\left(\frac{3 n}{3 k+1}-t\right)\right) \\
& >(k t+1) \times \frac{n}{k t+1}=n,
\end{aligned}
$$

a contradiction.

Remark 3.5. Now, we explain that the condition of toughness $\tau(G) \geq \frac{1}{k}$ in Theorem 3.1 and minimum degree $\delta(G) \geq \max \left\{\frac{n}{k+1}, \frac{4 n}{3 k+1}-1\right\}$ in Theorem 3.3 are all the best possible. Let $H_{1}, H_{2}, \ldots, H_{k+1}$ be $k+1$ odd complete 
graphs, each of which contains exactly $\frac{n-1}{k+1}$ vertices, where integer $k \geq 2$ and $\frac{n-1}{k+1}$ is an integer. We construct a connected graph $G=K_{1} \vee\left(\bigcup_{i=1}^{k+1} H_{i}\right)$, the order of which is $n$. It is obviously that $\tau(G)=\frac{1}{k+1}<\frac{1}{k}$, and $\delta(G)=\frac{n-1}{k+1}<\frac{n}{k+1}$. Choose $X=: V\left(K_{1}\right)$ with $|X|=1$, then we have that

$$
o c(G-X)=k+1>k|X|=k .
$$

It follows from Theorem 1.4 that $G$ has no $\mathcal{I} \mathcal{S}_{k}$-factor.

\section{PAth-FACTOR}

In this section, we obtain some sufficient conditions for the existence of graphs admitting $P_{\geq 3}$-factors.

The binding number is introduced by Woodall [12] and defined as

$$
\operatorname{bind}(G)=\min \left\{\frac{\left|N_{G}(S)\right|}{|S|}: \emptyset \neq S \subseteq V(G), N_{G}(S) \neq V(G)\right\} .
$$

Theorem 4.1. Let $G$ be a connected graph of order $n \geq 3$. Then $G$ has a $P_{\geq 3}$-factor if one of the following statements holds:

(i) $I(G) \geq \frac{3}{2}$;

(ii) $\operatorname{bind}(G) \geq \frac{5}{4}$;

(iii) $n \geq 8$ and for all three independent vertices $u, v, w \in V(G)$,

$$
\max \left\{d_{G}(u), d_{G}(v), d_{G}(w)\right\} \geq \frac{n}{3} .
$$

Proof. By way of contradiction, suppose that $G$ is a connected graph with no $P_{\geq 3}$-factor. Then by Theorem 1.5 , there is a subset $S \subseteq V(G)$ such that $\operatorname{sun}(G-S)>2|S|$. Due to the integrality of $\operatorname{sun}(G-S)$, we obtain

$$
\operatorname{sun}(G-S) \geq 2|S|+1 .
$$

(i) Obviously $G$ has a $P_{\geq 3}$-factor if $G$ is complete, a contradiction. Thus, we may assume that $G$ is not complete. We shall consider two cases by the value of $|S|$ and derive a contradiction in each case.

Case 1. $|S|=0$.

By (4.1), we have $\operatorname{sun}(G)=\operatorname{sun}(G-S) \geq 2|S|+1=1$. Note that $\operatorname{sun}(G) \leq \omega(G)=1$ since $G$ is connected. Then, $\operatorname{sun}(G)=1$ and thus $G$ is a big sun. Of course, $G$ is not an isolated edge since its order at least three. Let $R$ be the factor-critical subgraph of $G$ and set $U=V(R)$. It is clear that $G-U$ is an independent set and $|G-U|=|U|$. By the definition of $I(G)$ and $I(G) \geq \frac{3}{2}$, we have that

$$
\frac{3}{2} \leq I(G) \leq \frac{|U|}{i(G-U)}=1
$$

a contradiction.

Case 2. $|S| \geq 1$.

By (4.1), we have that

$$
|S| \leq \frac{\operatorname{sun}(G-S)-1}{2} .
$$

Assume that $\operatorname{sun}(G-S)-i(G-S)=m$, i.e., there are $m$ big sun components of $G-S$, denoted by $\mathcal{C}=$ $\left\{C_{1}, C_{2}, \ldots, C_{m}\right\}$. For each $i \in[1, m]$, let $R_{i}$ be the factor-critical subgraph of $C_{i}$ if $C_{i}$ is not an isolated edge, 
and choose vertices $c_{i} \in V\left(R_{i}\right)$. If $C_{i}$ is an isolated edge, then choose arbitrarily $c_{i} \in V\left(R_{i}\right)$ where $1 \leq i \leq m$. Let $S^{\prime}=\left\{c_{i}: 1 \leq i \leq m\right\}$. Then by (4.2), we have that

$$
\begin{aligned}
\left|S \cup S^{\prime}\right| & =|S|+\operatorname{sun}(G-S)-i(G-S) \\
& \leq|S|+\operatorname{sun}(G-S) \\
& \leq \frac{\operatorname{sun}(G-S)-1}{2}+\operatorname{sun}(G-S) \\
& =\frac{3 \times \operatorname{sun}(G-S)-1}{2} .
\end{aligned}
$$

By the definition of $I(G)$, it follows that

$$
\begin{aligned}
\frac{3}{2} \leq I(G) & \leq \frac{\left|S \cup S^{\prime}\right|}{i\left(G-S-S^{\prime}\right)} \\
& \leq \frac{3 \times \operatorname{sun}(G-S)-1}{2 \times i\left(G-S-S^{\prime}\right)} \\
& =\frac{3 \times \operatorname{sun}(G-S)-1}{2 \times \operatorname{sun}(G-S)}<\frac{3}{2},
\end{aligned}
$$

a contradiction.

The statement (i) in Theorem 4.1 is proved.

(ii) Let $S^{\prime}=V(G-S)$. By the definition of $\operatorname{bind}(G)$, we have that

$$
\left|N_{G}\left(S^{\prime}\right)\right| \geq \frac{5}{4}\left|S^{\prime}\right|
$$

Case 1. $|S| \geq \frac{n}{5}$.

In this case, $\left|S^{\prime}\right|=|G|-|S| \leq \frac{4 n}{5}$. By (4.3),

$$
\begin{aligned}
\frac{5}{4}(n-|S|) & =\frac{5}{4}\left|S^{\prime}\right| \leq\left|N_{G}\left(S^{\prime}\right)\right| \\
& =\left|N_{G}(G-S)\right| \leq n-i(G-S) .
\end{aligned}
$$

It follows immediately that

$$
i(G-S) \leq \frac{5}{4}|S|-\frac{n}{4}
$$

Hence, by (4.4),

$$
\begin{aligned}
n & \geq|S|+i(G-S)+2 \times(\operatorname{sun}(G-S)-i(G-S)) \\
& =|S|+2 \times \operatorname{sun}(G-S)-i(G-S) \\
& >|S|+4|S|-\left(\frac{5}{4}|S|-\frac{n}{4}\right) \\
& =\frac{15}{4}|S|+\frac{n}{4} \geq n,
\end{aligned}
$$

a contradiction. 
Case 2. $|S|<\frac{n}{5}$.

In this case, $\left|S^{\prime}\right|=|G|-|S|>\frac{4 n}{5}$. Let $S_{0} \subseteq S^{\prime}$ such that $\left|S_{0}\right|=\frac{4 n}{5}$. By (4.3), we have that $\left|N_{G}\left(S_{0}\right)\right| \geq$ $\frac{5}{4}\left|S_{0}\right|=n$ and so $V(G) \subseteq N_{G}\left(S^{\prime}\right)$. Consequently, there exists no singleton component of $G-S$, i.e.,

$$
i(G-S)=0 .
$$

Consider all the sun components in $G-S$ and let $S^{\prime \prime}=V(\operatorname{Sun}(G-S))$. Since $\operatorname{sun}(G-S)>2|S|$, by $(4.5)$, $\left|S^{\prime \prime}\right|>2 \times \operatorname{sun}(G-S)>4|S|$. Hence,

$$
\begin{aligned}
\operatorname{bind}(G) & \leq \frac{\left|N_{G}\left(S^{\prime \prime}\right)\right|}{\left|S^{\prime \prime}\right|} \leq \frac{\left|S^{\prime \prime}\right|+|S|}{\left|S^{\prime \prime}\right|} \\
& =1+\frac{|S|}{\left|S^{\prime \prime}\right|}<1+\frac{1}{4}=\frac{5}{4}
\end{aligned}
$$

a contradiction.

The statement (ii) in Theorem 4.1 is proved.

(iii) We first give the argument as following.

Claim 4.2. $S \neq \emptyset$.

Proof. Suppose $S=\emptyset$, by $(4.1), \operatorname{sun}(G)=\operatorname{sun}(G-S) \geq 1$. On the other hand, $\operatorname{sun}(G) \leq \omega(G)=1$. So, we obtain that $G$ is a big sun containing at least 8 vertices. It follows that there exist three vertices of degree one, denoted by $\{u, v, w\}$, which contradicts that $\max \left\{d_{G}(u), d_{G}(v), d_{G}(w)\right\} \geq \frac{n}{3}>2$.

By Claim 4.2 and (4.1), we have $\operatorname{sun}(G-S) \geq 2|S|+1 \geq 3$.

Case 1. $i(G-S) \geq 3$.

Let $\{x, y, z\}$ be three distinct isolated vertices of $G-S$. Since $\max \left\{d_{G}(x), d_{G}(y), d_{G}(z)\right\} \geq \frac{n}{3}$ and $N_{G}(x) \cup$ $N_{G}(y) \cup N_{G}(z) \subseteq S$, we have that

$$
|S| \geq \max \left\{d_{G}(x), d_{G}(y), d_{G}(z)\right\} \geq \frac{n}{3} .
$$

It follows from (4.1) that $\operatorname{sun}(G-S) \geq 2|S|+1 \geq \frac{2 n}{3}+1$ and thus

$$
n \geq|S|+\operatorname{sun}(G-S) \geq \frac{n}{3}+\frac{2 n}{3}+1=n+1,
$$

a contradiction.

Case 2. $i(G-S) \leq 2$.

In this case, by (4.1), there exist at least three suns of $G-S$, denoted by $C_{1}, C_{2}, \ldots, C_{t}$ where $t \geq 3$. Then we choose $c_{i} \in V\left(C_{i}\right)$ such that $d_{C_{i}}\left(c_{i}\right) \leq 1$, where $i=1,2,3$. Obviously, $\left\{c_{1}, c_{2}, c_{3}\right\}$ is an independent set of $G$. Then $\max \left\{d_{G}\left(c_{1}\right), d_{G}\left(c_{2}\right), d_{G}\left(c_{3}\right)\right\} \geq \frac{n}{3}$. Without loss of generality, we assume $d_{G}\left(c_{1}\right) \geq \frac{n}{3}$. Since $d_{S}\left(c_{1}\right)=d_{G}\left(c_{1}\right)-d_{C_{1}}\left(c_{1}\right) \geq \frac{n}{3}-1$, we have that $|S| \geq d_{S}\left(c_{1}\right) \geq \frac{n}{3}-1$. It follows from (4.1) that

$$
\operatorname{sun}(G-S) \geq 2|S|+1 \geq \frac{2 n}{3}-1,
$$

and thus

$$
\begin{aligned}
n & \geq|S|+2 \times \operatorname{sun}(G-S)-i(G-S) \\
& \geq \frac{n}{3}-1+2 \times\left(\frac{2 n}{3}-1\right)-2 \\
& =\frac{5 n}{3}-5>n,
\end{aligned}
$$

a contradiction. The statement (iii) in Theorem 4.1 is proved. 
Remark 4.3. Now, we claim that the conditions of isolated toughness $I(G) \geq \frac{2}{3}$ and binding number $\operatorname{bind}(G) \geq$ $\frac{5}{4}$ in Theorem 4.1 are all the best possible. Let $P_{5}$ be a path of order 5 , the center vertex of which is denoted by $u$. We construct a connected graph $G=P_{5} \cup\{v\} \cup e$, where $e=u v$. It is obvious that $I(G)=1<\frac{3}{2}$, and $\operatorname{bind}(G)=1<\frac{5}{4}$. Choose $X=:\{u\}$, then we have that $\operatorname{sun}(G-X)=3>2=2|X|$. It follows from Theorem 1.5 that $G$ has no $P_{\geq 3}$-factor.

Remark 4.4. Now, we explain that the degree condition in the statement (iii) of Theorem 4.1 is the best possible. Let $G=2 K_{1} \vee 7 K_{1}$ be a connected complete bipartite graph of order $n=9$. We know there exists three independent vertices $\{u, v, w\} \subseteq V\left(7 K_{1}\right)$ such that $\max \left\{d_{G}(u), d_{G}(v), d_{G}(w)\right\}=2<3=\frac{n}{3}$. Choose $X=: V\left(2 K_{1}\right)$ with $|X|=2$, then we have that $\operatorname{sun}(G-X)=7>2|X|=4$. Using Theorem $1.5, G$ has no $P_{\geq 3}$-factor.

Acknowledgements. The authors are indebted to the anonymous referees for their kind comments and constructive suggestions in improving this paper. This work was supported by the National Natural Science Foundation of China (Grant Nos. 11871239 and 11971196)

\section{REFERENCES}

[1] J. Akiyama, D. Avis and H. Era, On a \{1,2\}-factor of a graph. TRU Math. 16 (1980) 97-102.

[2] A. Amahashi and M. Kano, Factors with given components. Discrete Math. 42 (1982) 1-6.

[3] J.A. Bondy and U.S.R. Murty, Graph theory with applications. North-Holland, New York-Amsterdam-Oxford (1982).

[4] V. Chvátal, Tough graphs and Hamiltonian Circuits. Discrete Math. 5 (1973) 215-228.

[5] G. Dai, The existence of path-factor covered graphs. To appear in: Discuss. Math. Graph Theory (2020).

[6] Y. Egawa, M. Kano and A.K. Kelmans, Star partitions of graphs. J. Graph Theory 25 (1997) 185-190.

[7] A. Kaneko, A necessary and sufficient condition for the existence of a path factor every component of which is a path of length at least two. J. Combin. Theory Ser. B 88 (2003) 195-218.

[8] M. Kano, G.Y. Katona and Z. Király, Packing paths of length at least two. Discrete Math. 283 (2004) $129-135$.

[9] M.D. Plummer, Perspectives: Graph factors and factorization: 1985-2003: A survey. Discrete Math. 307 (2007) $791-821$.

[10] W.T. Tutte, The factors of graphs. Canad. J. Math. 4 (1952) 314-328.

[11] M. Las Vergnas, An extension of Tutte's 1-factor theorem. Discrete Math. 23 (1978) 241-255.

[12] D.R. Woodall, The binding number of a graph and its Anderson number. J. Combin. Theory Ser. B 15 (1973) $225-255$.

[13] Q. Yu and G. Liu, Graph Factors and Matching Extensions. Higher Education Press, Beijing (2009).

[14] S. Zhou, Some results about component factors in graphs. RAIRO-Oper. Res. 53 (2019) 723-730.

[15] S. Zhou, Q. Bian and Z. Sun, Two sufficient conditions for component factors in graphs. To appear in: Discuss. Math. Graph Theory (2021).

\section{Subscribe to Open (S2O) A fair and sustainable open access model}

This journal is currently published in open access under a Subscribe-to-Open model (S2O). S2O is a transformative model that aims to move subscription journals to open access. Open access is the free, immediate, online availability of research articles combined with the rights to use these articles fully in the digital environment. We are thankful to our subscribers and sponsors for making it possible to publish this journal in open access, free of charge for authors.

\section{Please help to maintain this journal in open access!}

Check that your library subscribes to the journal, or make a personal donation to the S2O programme, by contacting subscribers@edpsciences.org

More information, including a list of sponsors and a financial transparency report, available at: https://www. edpsciences.org/en/maths-s2o-programme 